\title{
Species Differences in Reactivity of Mouse and Rat Astrocytes in vitro
}

\author{
Till B. Puschmann Kirsty J. Dixon Ann M. Turnley \\ Centre for Neuroscience, The University of Melbourne, Melbourne, Vic., Australia
}

\author{
Key Words \\ Astrocytic gliosis • Glial fibrillary acidic protein • Vimentin • \\ Scratch wound $\cdot$ Cytosine $\beta$-D-arabinofuranoside \\ hydrochloride
}

\begin{abstract}
Reactive astrogliosis constitutes a major obstacle to neuronal regeneration and is characterized by rearrangement and upregulation of expression of cytoskeletal proteins, increased proliferation and hypertrophy. Many approaches have been attempted to mimic astrogliosis by inducing reactive astrocytes in vitro. Such research is usually performed using astrocytes derived from Mus musculus or Rattus norvegicus, and results compared between species on the assumption that these cells behave equivalently. Therefore, we compared reactivity between mouse and rat astrocytes in scratch wound assays to gain further insight into how comparable these cell culture models are. Proliferation and migration, as well as expression of the cytoskeletal proteins glial fibrillary acidic protein (GFAP) and vimentin, were compared by immunocytochemistry and immunoblot. Further, we investigated migration of proliferating cells by 5-ethynyl2 '-deoxyuridine staining. Substantial differences in GFAP expression and proliferation between astrocytes of the two species were found: rat astrocytes showed different cytoskeletal morphology, expressed significantly more GFAP and vimentin of different molecular size and were more proliferative than comparable mouse astrocytes. Our results sug-
\end{abstract}

gest that rat and mouse astrocytes may respond differently to various reactivity-triggering stimuli, which needs to be considered when general conclusions are drawn regarding effects of factors regulating astrocyte reactivity.

Copyright $\odot 2010$ S. Karger AG, Basel

\section{Introduction}

Following central nervous system (CNS) injury, astrocytes become reactive, proliferate and undergo hypertrophy [1], leading to glial scar formation at the injury site [2, $3]$. Reactive astrocytes undergo cytoskeletal and hence, morphological changes [4]. Upregulation of the expression of a key molecule, the astrocyte marker glial fibrillary acidic protein (GFAP), is commonly used to characterize astrocyte reactivity in vivo [5]. However, many biological processes involving astrogliosis are difficult to study in vivo. The large number of different cell types in the CNS poses a complicated problem for identification of primary effects of single cell types or secondary effects of other cell-signaling pathways involved. Astrocyte cultures are useful research tools and several models of astrogliosis have been used to study the process of astrocyte activation in vitro.

Approaches to induce reactivity in culture were first attempted by chemical treatment. Dibutyryl cAMP and noradrenaline were shown to regulate astrocyte morphology [6,7] and had been believed to trigger astrocyte

\section{KARGER \\ Fax +41613061234 \\ E-Mail karger@karger.ch}

www.karger.com
(C) 2010 S. Karger AG, Basel

$1424-862 X / 10 / 0183-0152 \$ 26.00 / 0$

Accessible online at:

www.karger.com/nsg
Ann Turnley

Centre for Neuroscience

The University of Melbourne

Melbourne, VIC 3010 (Australia)

Tel. +61 38344 3981, E-Mail turnley@unimelb.edu.au 
reactivity in culture because cells changed from their typical polygonal shape to a star-shaped morphology, similar to the shape of reactive astrocytes in vivo [8]. Later research, however, showed that those cells appear merely to change their morphology but lack other properties of reactive astrocytes, such as the ability to inhibit neurite outgrowth [9]. A large amount of research has been conducted on astrocytic monolayers. In these experiments, in vitro cultures are activated by scratching confluent monolayers with needles, blades or pipette tips $[10,11]$. The mechanical stress on astrocytes and the partial removal of cell-cell contacts lead to release of contact inhibition of proliferation. It has also been reported to lead to cytoskeletal changes, such as increases in nestin and GFAP protein expression, similar to what is observed in astrocytes following stab wound experiments in vivo $[12,13]$. Scratch wound assays have been used to investigate a range of effects on astrocytes under activated conditions, such as the influence of the extracellular matrix protein tenascin- $\mathrm{C}$ on cell migration and proliferation [14], the effects of gene silencing in reactive astrocytes [11], the role of ephrin receptor signaling of astrocyte cytoskeletal rearrangement [15] or the effects of soluble growth factors on wound healing [16]. Scratch wound assays have also been used in research using various cell types, such as smooth muscle cells [17] or corneal endothelial cells [18], demonstrating the importance of the method in investigating reactive cell behavior.

It has been shown that astrocytes in a scratch wound model react with increased proliferation, as measured by bromodeoxyuridine (BrdU) uptake [12], and it has further been suggested that regulating the cell cycle of reactive astrocytes by inhibiting proliferation could lead to a decrease in the production of signaling molecules involved in glial scar formation [19]. In high-density cultures, astrocytes exhibit homotypic cell contact inhibition of proliferation. After injury or insult in the scratch wound model, the cell-cell contact inhibition of proliferation is released and cells become hyperproliferative $[12,20]$. It has also been suggested that all astrocytes in culture are reactive, based on high levels of expression of the astrocyte marker GFAP. However, this is predominantly in the commonly used astrocyte cultures derived from neonatal animals. In contrast, cells from adults do not appear to have a reactive phenotype in culture $[21,22]$. Therefore, there are age-dependent differences in astrocyte reactivity in culture. However, whether similar differences exist between species has not yet been described. We show here that there are indeed large species differences between neonatal rat and mouse cortical astrocyte cultures. We found that levels of the cytoskeletal proteins GFAP and vimentin were increased in rat astrocytes under control and scratch wound conditions, and there were basal differences as well in percentage of astrocytes expressing GFAP, with much higher levels detected in rat cultures. Further, rat astrocytes were more proliferative under control conditions and after a scratch insult than mouse cells. Despite this increased proliferation, scratch closure speed and cell invasion appeared to be similar in rat and mouse astrocyte cultures. Therefore, although the same end result of scratch wound closure was obtained, the mechanisms leading to it differed between species.

\section{Material and Methods}

\section{Dissection of Mouse and Rat Cortices}

Animal use was approved by the University of Melbourne Animal Ethics Committee and experiments were performed in accordance with National Health and Medical Research Council of Australia guidelines. Brains from newborn (P1-P3) C57BL/6 mouse and Sprague-Dawley rat were simultaneously dissected under sterile conditions. The cortex was isolated and tissue samples were dissociated mechanically by chopping in Dulbecco's Modified Eagle Medium (DMEM; Millipore, Melbourne, Australia) with a sterile scalpel blade. The tissue was incubated for $20 \mathrm{~min}$ at $37^{\circ} \mathrm{C}$ in $1 \mathrm{mg}$ DNase1 (Sigma, Castle Hill, Australia) and $25 \mathrm{mg}$ Trypsin (Gibco, Melbourne, Australia) in $100 \mathrm{ml}$ HEPES-buffered Eagle's Medium. After 20 min, DMEM containing $10 \%$ fetal bovine serum (Thermo scientific, Australia) was added to stop trypsinization and cells were pelleted at $1,500 \mathrm{rpm}$ $(478 \mathrm{~g})$ for $5 \mathrm{~min}$. Pellets were resuspended in DMEM supplemented with $10 \%$ fetal calf serum, penicillin $(100 \mathrm{U} / \mathrm{ml}$, Invitrogen, Melbourne, Australia) and streptomycin $(100 \mu \mathrm{g} / \mathrm{ml}$, Invitrogen). At $24 \mathrm{~h}$ after plating, astrocyte cultures were shaken vigorously to remove nonadhesive nonastrocytic cells followed by two wash steps with phosphate-buffered saline (PBS) and medium replacement. To reduce microglial growth, the culture medium was changed every second day. The cultures routinely contained $>98 \%$ astrocytes, as assessed by expression of the astrocyte markers GLT1 and S100 $\beta$ (online supplementary figure 1, www. karger.com/doi/10.1159/000321494), <0.5\% microglia (online suppl. fig. 1) and $<0.1 \% \mathrm{O}_{4}$-positive oligodendrocytes and $\beta I I I-$ tubulin-positive neurons (data not shown).

\section{Astrocyte Plating and Scratch Wound Assays}

To achieve consistency between experiments, freshly dissected mouse and rat astrocytes were plated into T80 flasks at a density of $2 \times 10^{6}$ cells per T80 flask (Nunc, Roskilde, Denmark) and grown to confluence alongside each other for 10 days at $37^{\circ} \mathrm{C}$ in a humidified atmosphere containing $5 \% \mathrm{CO}_{2}$. Cells were then frozen or directly transferred into 24-well plates for scratch wound assays or 100-mm dishes (BD Falcon, North Ryde, Australia) for Western blot analysis.

All cell culture vessels were coated for $4 \mathrm{~h}$ with poly-D-lysine (10 $\mu \mathrm{g} / \mathrm{ml}$; Sigma) in double-distilled $\mathrm{H}_{2} \mathrm{O}$, then vessels were washed 3 times with double-distilled $\mathrm{H}_{2} \mathrm{O}$ before cells were seed- 
ed in cell culture medium $\left(3 \times 10^{4}\right.$ cells/well in 24 -well dishes for scratch wound experiments and $1 \times 10^{6}$ cells per $100-\mathrm{mm}$ dish for Western blot experiments).

After 4-6 days in 24-well plates, astrocyte monolayers were scratched with a sterile 20 - to $200-\mu l$ plastic pipette tip (Axygen, Union City, Calif., USA). This created a cell-free area of approximately 500-600 $\mu \mathrm{m}$. Cells were washed twice with sterile PBS, and medium was replaced. After $24,48 \mathrm{~h}$ and $72 \mathrm{~h}$ of incubation, during which time the medium was not exchanged, cells were fixed with $4 \%$ paraformaldehyde prior to immunocytochemistry.

In some experiments, cell proliferation was inhibited by addition of $20 \mu \mathrm{M}$ cytosine $\beta$-D-arabinofuranoside hydrochloride (AraC, Sigma), starting from $24 \mathrm{~h}$ prior to scratching to $72 \mathrm{~h}$ following scratching. In those experiments, the percent of proliferative cells was measured by addition of the BrdU analogue EdU to the cultures $3 \mathrm{~h}$ before cell fixation, according to the manufacturer's instructions (Invitrogen).

\section{Immunocytochemistry and Cell Counts}

After fixation in $4 \%$ paraformaldehyde, cells were permeabilized with $0.1 \%$ Triton X-100 in PBS, then blocked in PBS containing $2 \%$ fetal calf serum and $2 \%$ goat serum (blocking solution). After blocking, cells were incubated for $30 \mathrm{~min}$ with primary antibodies diluted in blocking solution: mouse anti-GFAP (1:1,000, Sigma), rabbit anti-GFAP (1:1,000, Dako, Denmark), guinea pig anti-GLT1 (1:2,000, Chemicon, Australia), mouse anti-S100 $\beta$ (1:500, Sigma), rat anti-CD11b (1:400 Millipore), rabbit anti-Ki67 (1:200, Labvision, Australia), mouse anti-vimentin (1:1,000, Chemicon). Cells were washed three times in PBS and incubated with appropriate secondary antibodies diluted in blocking solution: Alexa 488-conjugated donkey anti-rabbit (1:500, Invitrogen, Australia), Cy3-conjugated sheep anti-mouse IgG (1:1,000, Jackson ImmunoResearch Laboratories, USA), Cy2-conjugated goat anti-mouse IgG (1:1,000, Jackson ImmunoResarch Laboratories) and biotinylated donkey anti-guinea pig (1:500, Millipore) followed by Cy3-streptavidin (1:1,000, Millipore). The nuclear stain 4',6-diamidino-2-phenylindole (DAPI) was added along with the secondary antibodies. For EdU detection, the 'click-it' reaction was performed according to the manufacturer's instructions (Invitrogen), prior to addition of primary antibodies.

Photomicrographs of the scratched area and control areas $>250$ $\mu \mathrm{m}$ away from the border of the scratch were taken randomly. In preliminary experiments, cells in areas $>250 \mu \mathrm{m}$ away from the scratch were indistinguishable from cells in unscratched wells (data not shown); therefore, all cells within the scratch were compared to cells in control areas within the same well. The number of astrocytes entering the wound area was determined by counting the number of DAPI-positive nuclei found within the scratched areas. As $>98 \%$ of cells expressed the astrocyte markers GLT-1 or S100 $\beta$, there was very little contamination by nonastrocytic cells and all nuclei were counted as astrocytes. All quantitative analyses of GFAP immunohistochemistry reported herein were performed on cells labeled using the mouse anti-GFAP antibody, although the rabbit and mouse anti-GFAP antibody produced similar labeling (online suppl. fig. 1). The number of astrocytes in the control area was counted in a defined region of interest $\left(0.1 \mathrm{~mm}^{2}\right)$ from the center of each control picture and the number of cells positive for GFAP, vimentin and Ki67 expression was quantified. For measurements of the area covered by cytoskeletal proteins in cells expressing GFAP and vimentin, stained scratch wounds were ana- lyzed using NIH Image (Wayne Rasband, National Institutes of Health, USA). A threshold above background was set for each individual image and the pixel area above threshold was measured, followed by measurement of DAPI-positive cell numbers in each image and the percentage of cells that expressed the protein. Area values were divided by DAPI-positive cell numbers and multiplied by the percentage of cells that expressed each protein to obtain the area of GFAP or vimentin per cell.

\section{Western Blot Analysis}

Astrocytes were plated into poly-D-lysine-coated $100-\mathrm{mm}$ dishes and grown 3-4 days to confluence. Once cells were confluent, 6 horizontal and 6 vertical scratches per $100-\mathrm{mm}$ dish were done, followed by 3 washing steps and replacement of medium for $48 \mathrm{~h}$, as outlined in the scratch wound procedure description above. Cell lysis, immunoprecipitation and immunoblotting were performed according to standard protocols, as described in Puschmann and Turnley [15]. For biochemical analysis of astrocytes, protein was isolated from cultured cells by lysis in lysis buffer [20 mM Tris-HCl, pH7.5, $150 \mathrm{mM} \mathrm{NaCl}, 1 \mathrm{~mm}$ EDTA, $1 \mathrm{~mm}$ EGTA, $1 \%$ Triton X-100, $1 \mathrm{~mm}$ sodium $o$-vanadate, 1 tablet $/ 10 \mathrm{ml}$ phospho-stop (Roche, Australia), 1 tablet $/ 10 \mathrm{ml}$ protease inhibitor cocktail (Roche)] using a cell scraper. Lysates were then sonicated for $10 \mathrm{~s}$ and either snap-frozen in liquid nitrogen or used instantly. Protein concentration was measured using a standard Bradford assay (Bio-Rad, Melbourne, Australia) so that equal amounts of lysate could be loaded for each experimental condition.

Proteins $(20 \mu \mathrm{g})$ were loaded onto $12 \%$ SDS-PAGE gels (NuSep, Sydney, Australia) and then transferred to nitrocellulose membrane (Bio-Rad). Membranes were blocked in 5\% bovine serum albumin (Sigma) in Tris-buffered saline (TBS) containing $0.1 \%$ Tween-20 (TBST) for $45 \mathrm{~min}$, then primary antibody was added and incubated overnight at $4^{\circ} \mathrm{C}$. Primary antibodies used were rabbit anti-GFAP (1:2,000, Dako, Denmark), mouse anti-vimentin (1:1,000, Millipore) and mouse anti-GAPDH (1:5,000, Millipore). Membranes were then washed 3 times in TBST for $10 \mathrm{~min}$ and incubated with secondary antibodies, horseradish peroxidase-antimouse (1:10,000, Millipore) or horseradish peroxidase-anti-rabbit (1:10,000, Millipore) for $45 \mathrm{~min}$ at room temperature. After washing 3 times in TBST for $5 \mathrm{~min}$, the membranes were exposed to ECL solution (Amersham, Australia) and then scanned using gel documentation equipment (LAS3000, Fuji, Japan) and software (MultiGauge, Fuji, Japan). Membranes were then stripped using stripping buffer (Millipore) and reprobed for GAPDH.

\section{Statistical Analysis}

All results are presented as means \pm SEM from 3-5 combined experiments. Group data were analyzed by ANOVA, followed by Bonferroni post-hoc comparisons, using GraphPad Prism 4.0 software. Significance was set as $\mathrm{p}<0.05$.

\section{Results}

To investigate differences between mouse and rat astrocyte responses to reactivity-triggering stimuli in vitro, we conducted scratch wound assays on astrocyte monolayers and analyzed changes in cytoskeletal protein ex- 
pression and proliferation. At 24,48 and $72 \mathrm{~h}$ after scratch, cells were fixed and immunostained for the mature astrocyte marker, GFAP, the immature/reactive astrocyte marker, vimentin, and the proliferative cell marker, Ki67. All cultures were also stained with the nuclear label, DAPI (fig. 1). By $72 \mathrm{~h}$, the scratch wound was largely closed by cells of both species.

\section{Rat Astrocytes Are More Proliferative than Mouse Astrocytes}

DAPI counts of astrocytes invading the scratch revealed that, although the numbers of cells invading the scratch increased over $72 \mathrm{~h}$ for both species, there were significantly higher numbers of rat astrocytes within the scratches at all time points, compared with mouse astrocytes (fig. 2a). Immunostaining for the proliferation marker Ki67 showed that, within the first $48 \mathrm{~h}$ after scratch, the percentage of proliferative astrocytes was higher in rat than in mouse astrocytes in control areas (fig. 2b). Rat cells within the scratch showed significantly higher percentages of Ki67-positive cells compared with mouse cells at all time points. Comparison between cells in control areas and cells within the scratch showed more proliferative rat cells within the scratch at all time points whereas mouse astrocytes only showed enhanced proliferation at $48 \mathrm{~h}$. This indicated that in the in vitro scratch wound assay, rat astrocytes reacted with an increased proliferative response compared with mouse cells. In control areas, the observed decline of Ki67 expression over time was likely caused by the increased density of the cultures, leading to enhanced cell-cell contact-mediated inhibition of proliferation.

Rat and Mouse Astrocytes Express Different Levels of GFAP

Analysis of GFAP immunocytochemistry in figure 1 indicated that rat cultures expressed more GFAP than mouse cultures, which was confirmed by Western blot analysis for GFAP. No differences in the level of GFAP expression could be detected between control and scratched cultures (fig. 3a). To assess whether the comparatively higher GFAP expression levels in rat astrocytes were due to higher levels of GFAP expression per cell or to a greater number of cells expressing GFAP, the percentage of astrocytes positive for GFAP within the scratch and in control areas was quantified. At all time points, a greater percentage of rat astrocytes expressed GFAP, both in control areas and within the scratch (fig. 3b), indicating that the higher level of GFAP expression detected by Western analysis was likely primarily due to the increased

Differences in Mouse and Rat Astrocyte Reactivity numbers of rat astrocytes expressing the protein, although they may also have higher levels per cell.

\section{Rat and Mouse Astrocytes Express Different Levels of Vimentin}

Similar to GFAP expression, Western blot analysis indicated higher levels of vimentin expression in rat compared with mouse astrocytes (fig. 3c). Again, no difference between scratched and control cultures could be detected. However, cell counts for vimentin-positive cells within the scratch and in control areas revealed no significant differences in percentages of cells expressing vimentin between the two cell types. Almost all cells of both species were vimentin positive (fig. $3 \mathrm{~d}$ ). This finding indicates that the higher level of vimentin expression in the rat cultures is due to higher levels of protein expression per cell.

\section{Rat and Mouse Astrocytes Show Morphological}

Differences in Response to Scratch Wound

At $24 \mathrm{~h}$ after scratch, most rat astrocytes at the border of the scratch had developed long, unipolar, GFAP-filled cell protrusions pointing from the soma into the scratch (fig. 3e). Mouse astrocytes in general lacked such long GFAP-filled protrusions. The morphology of mouse astrocytes was more polygonal, with smaller protrusions than those of rat cells, and the protrusions were less directed toward the scratch (fig. 3e). However, many astrocytes within the scratch lacked GFAP expression and this was observed for both rat and mouse astrocytes (fig. 1, $3 \mathrm{e})$. This is in contrast to vimentin, where almost all astrocytes of both species were vimentin positive. Vimentin expression increased in the majority of astrocytes that were at the border of and within the scratch (fig. 1, 3e). Again, rat astrocytes showed long thin, vimentin-filled protrusions pointing towards the scratch, while mouse astrocytes appeared to have rounder somas and a larger number of short protrusions. The orientation of the mouse vimentin-filled protrusions was less organized, with no obvious direction towards the scratch. Rat astrocytes changed the direction of their vimentin-containing filopodia to parallel to the scratch after they had bridged the scratched area at $48 \mathrm{~h}$ (fig. 1, 3e).

\section{Scratch Wound Closure}

Given the differences in expression of GFAP and vimentin, we were interested in determining whether there were species differences in the ability of GFAP-expressing and vimentin-expressing astrocytes to repair the scratch. We expressed this as the area of the scratch cov- 


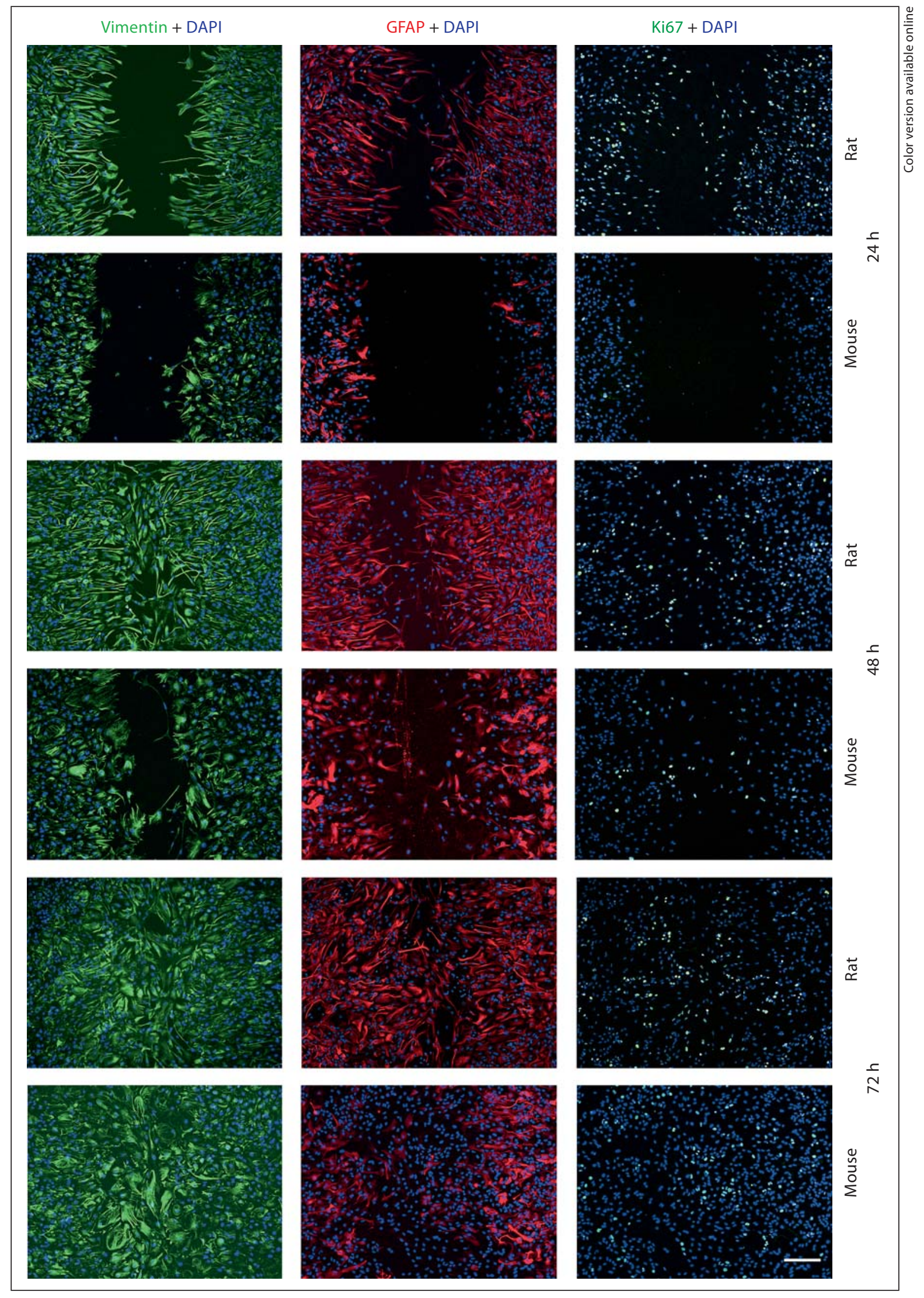




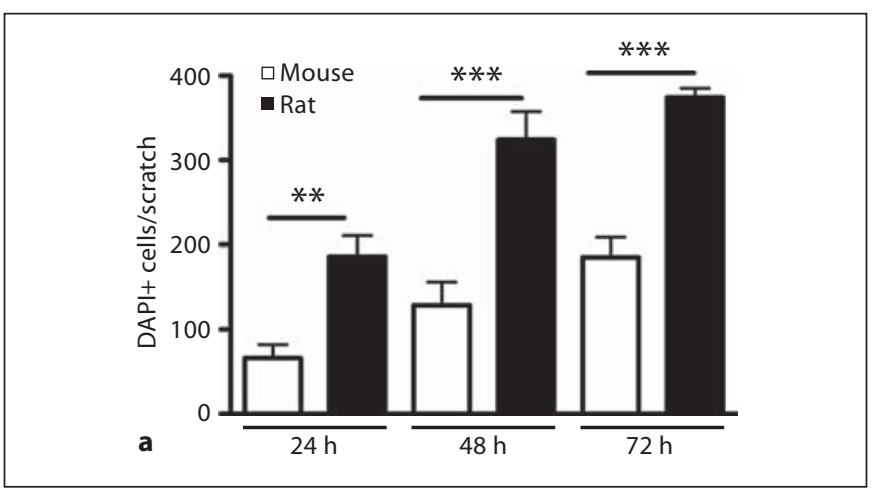

Fig. 2. Rat astrocytes were more proliferative and had increased numbers of cells present in the scratch at all time points. a Quantitation of the number of DAPI-stained nuclei in the scratch area indicated that there were increased numbers of rat astrocytes present in the scratch at all time points examined. $\mathbf{b}$ The number

ered by GFAP or vimentin at 24, 48 and $72 \mathrm{~h}$ using thresholding in image analysis to delineate the GFAP- and vimentin-covered areas. At all time points, GFAP-expressing rat astrocytes covered a significantly larger area of the scratch than mouse cells, while vimentin-expressing rat cells covered a larger area than mouse cells until $48 \mathrm{~h}$, but by $72 \mathrm{~h}$ there was no significant difference between species (fig. 4a). Further, for mouse astrocytes, the area covered by GFAP, compared with vimentin, was significantly smaller at all time points. In rat cells, this difference lasted only for the first $48 \mathrm{~h}$ after scratching (fig. $4 \mathrm{a}$ ). Figure $4 \mathrm{a}$ indicates that maximally only approximately $55 \%$ of the area was covered by vimentin or GFAP, even though at $72 \mathrm{~h}$ the scratches were mostly closed by cells (fig. 1).

Fig. 1. Scratch wound closure is faster in rat than mouse astrocyte cultures and vimentin-expressing cells respond more quickly. Rat and mouse astrocyte monolayers were scratched and immunostained for the astrocyte markers vimentin and GFAP (using mouse anti-GFAP) or the proliferation marker Ki67 at 24, 48 and $72 \mathrm{~h}$ after scratching. All cultures were also stained with the nuclear marker DAPI. Mouse cells were slower than rat cells to close the wound, but in both species the wound was closed by $72 \mathrm{~h}$. Scale bar: $200 \mu \mathrm{m}$.

Differences in Mouse and Rat Astrocyte Reactivity

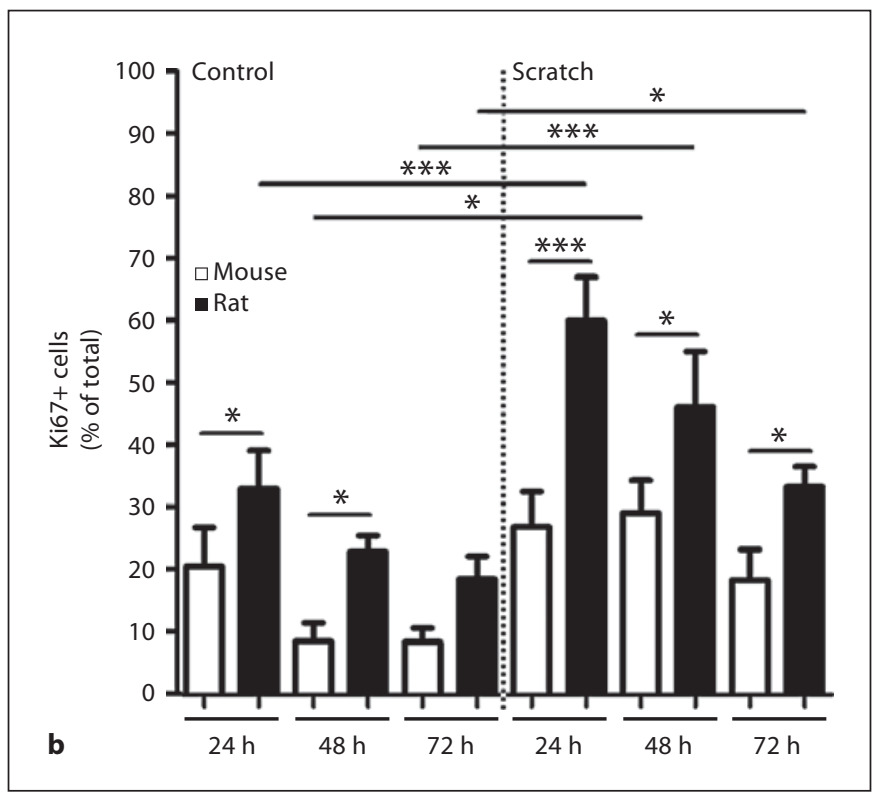

of proliferative, Ki67-positive cells was counted at each time point and presented as a percentage of total cell number. Rat astrocytes were more proliferative than mouse astrocytes. Results are means \pm SEM from 5 (a) and 4 (b) experiments; ${ }^{*} \mathrm{p}<0.05,{ }^{*} \mathrm{p}<0.01$, *** $\mathrm{p}<0.001$.

This is because only the area of GFAP or vimentin protein was measured and not the total area covered by the cells. To link the area covered by cytoskeletal proteins to cell numbers, the average area covered by each of the cytoskeletal proteins was divided by the fraction of cells in the scratch that expressed GFAP or vimentin. This indicated that GFAP and vimentin expression in individual mouse cells covered a larger area per cell compared with rat cells, suggesting a larger cell size (fig. $4 \mathrm{~b}, \mathrm{c}$ ), which could explain why, despite increased rat cell numbers within the scratch, mouse and rat astrocytes both closed a scratch wound within $72 \mathrm{~h}$.

\section{Scratch Wound Cell Invasion Is Similar in Rat and} Mouse Astrocytes when Proliferation Is Inhibited

To examine whether the faster scratch closure by rat astrocytes (fig. 1) was primarily due to the increased proliferation observed in these cells or whether increased cell migration was also involved, cell proliferation was blocked by addition of AraC $24 \mathrm{~h}$ before and during scratch wound closure. In contrast to control conditions, after inhibition of proliferation no significant differences in the total number of DAPI-stained cells that were present in the scratch could be detected between rat and 


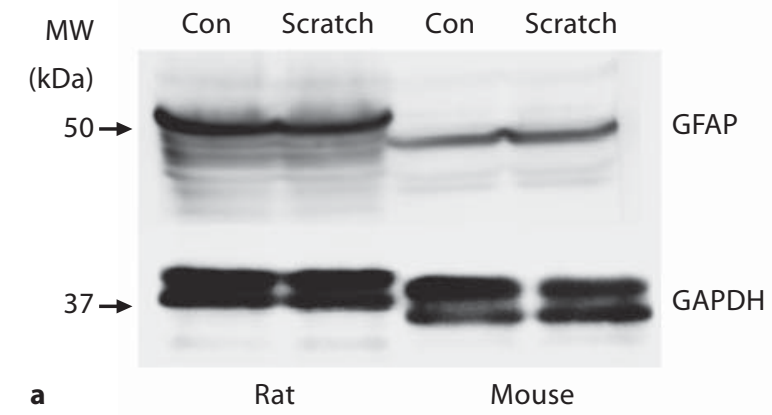

a
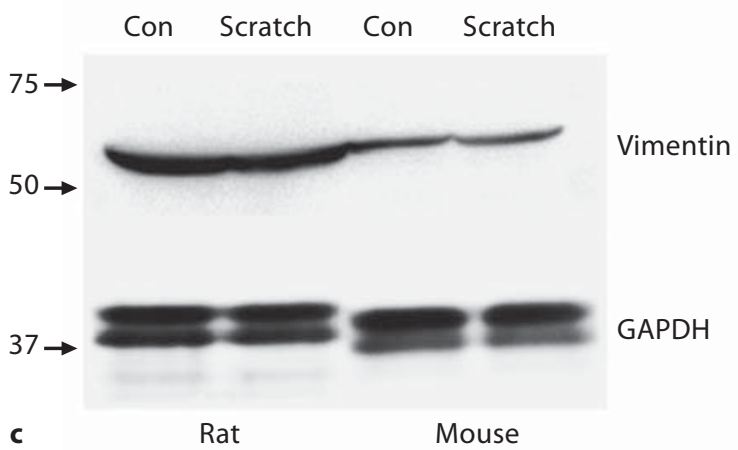

GFAP
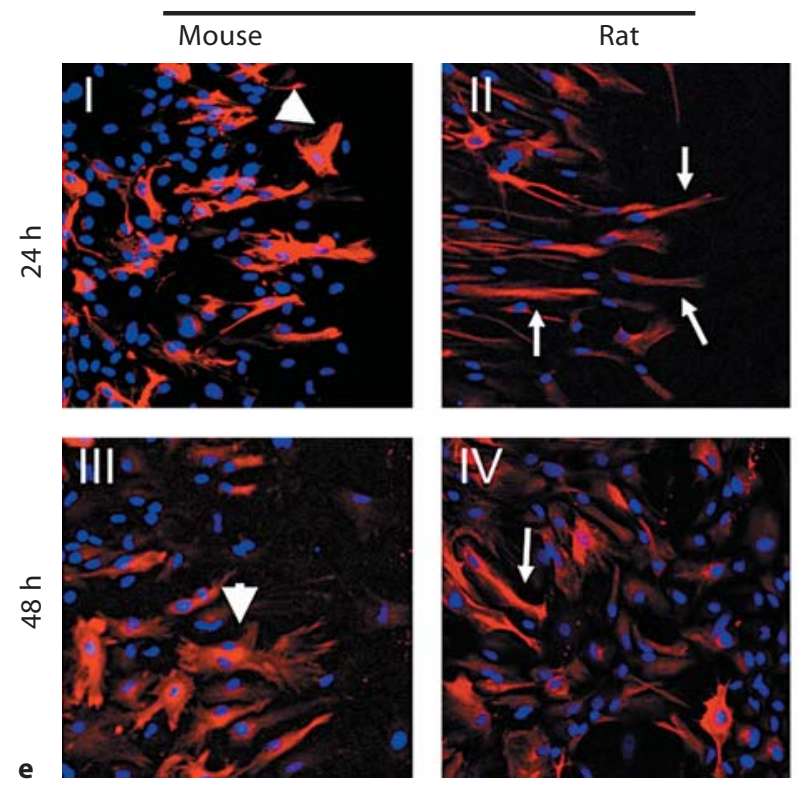

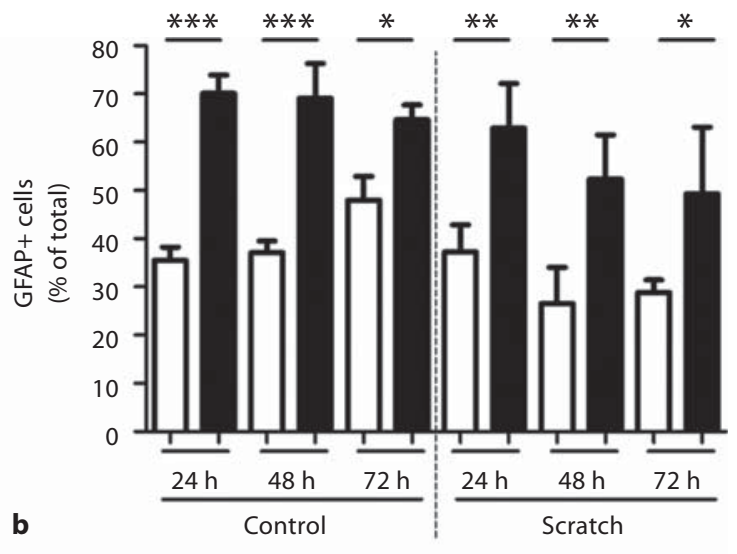

$\square$ Mouse

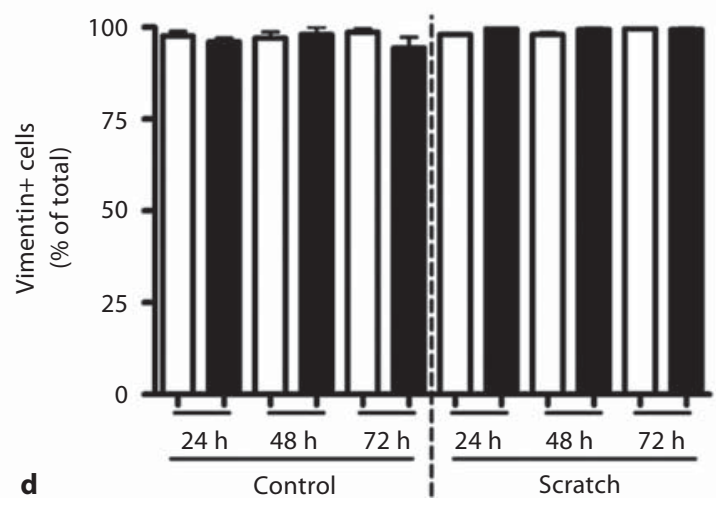

d

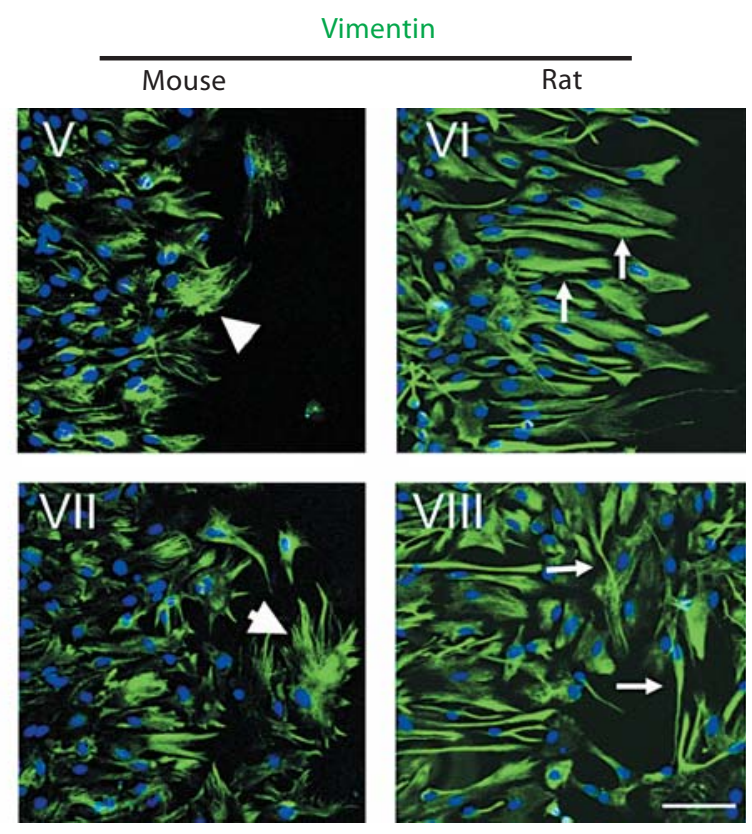

3 
mouse astrocytes (fig. 5a). This was primarily due to an effect on rat astrocytes, with significantly decreased numbers of rat astrocytes present in the scratch after AraC treatment at all time points. Significantly decreased numbers of mouse astrocytes following AraC treatment were only detected at $72 \mathrm{~h}$ after scratch.

When scratch wound closure by assessment of area covered by GFAP or vimentin was examined, there was little difference between rat and mouse astrocytes after inhibition of proliferation (fig. 5b). For the first $24 \mathrm{~h}$, the area of scratch closure was similar to normal proliferative conditions (fig. 4a) and the area covered by mouse GFAP was significantly less than that covered by rat GFAP. This was presumably due to the increased numbers of rat astrocytes expressing GFAP (fig. 3b). There was no further increase in area covered by GFAP or vimentin after the first $24 \mathrm{~h}$, indicating that proliferation of vimentin-positive cells in both species contributed to scratch wound closure. However, AraC treatment had little effect on mouse GFAP-expressing cells, which covered approximately the same area as non-AraC-treated cultures, while the area covered by rat GFAP-expressing cells was significantly inhibited (fig. 4a, 5b). This indicated that there are differences in the proliferation of rat and mouse GFAP-expressing cells and that the increased scratch closure speed of rat GFAP-expressing astrocytes in control, non-AraC-treated conditions is primarily due to increased proliferation, rather than migration.

Fig. 3. Comparison of GFAP and vimentin expression in rat and mouse astrocytes. a Western blot analysis of GFAP expression in control and scratched astrocyte cultures showed that GFAP was expressed at higher levels in rat cultures and exhibited a slightly higher molecular weight than mouse GFAP. No upregulation of GFAP could be detected in the scratched cultures. b The percentage of rat astrocytes that expressed GFAP was higher than that of mouse astrocytes under control and scratched conditions, at all time points tested. c Western blot analysis of vimentin expression in control and scratched astrocyte cultures showed that vimentin was expressed at higher levels in rat cultures and had a slightly lower molecular weight than mouse vimentin. No upregulation of vimentin could be detected in the scratched cultures. $\mathbf{d}$ Almost all cells in rat and mouse cultures expressed vimentin in control and scratched areas. e Immunostaining of mouse and rat cultures at 24 and $48 \mathrm{~h}$ revealed morphological differences between rat and mouse cells. Arrows in panels II, IV and VI indicate large processes in rat astrocytes oriented toward the lesion site and in panel VIII, parallel to the lesion. Arrowheads in mouse cultures in panels I, III, V and VII indicate cells with nonoriented processes. Cells were costained with DAPI (blue). Scale bar: $100 \mu \mathrm{m}$. Results in $\mathbf{b}$ and $\mathbf{d}$ are means \pm SEM from 3 experiments; ${ }^{*} \mathrm{p}<0.05$, ${ }^{* *} \mathrm{p}<0.01,{ }^{* * *} \mathrm{p}<0.001$.

Differences in Mouse and Rat Astrocyte Reactivity
To further investigate whether there was a correlation between proliferating and migrating astrocytes, cultures were incubated with EdU for $3 \mathrm{~h}$, either before monolayers were scratched or $45 \mathrm{~h}$ after monolayers were scratched, to label cells in cycle. For cultures labeled $3 \mathrm{~h}$ prior to scratch, within the first $24 \mathrm{~h}$, a significantly larger percentage of rat cells than mouse cells within the scratch were labeled with EdU in control conditions and this was ablated by the addition of AraC (fig. 5c). From $48 \mathrm{~h}$ there were no significant differences in the percentage of EdU-positive cells between control or AraC-treated mouse or rat cells (fig. 5c) and the number of EdUpositive cells per scratch did not increase over time (data not shown). This indicates that the large increase in total cell numbers in the scratch under control conditions at 48 and $72 \mathrm{~h}$ (fig. 5a) was largely derived from cells that were nonproliferative prior to scratching. Figure $5 \mathrm{e} \mathrm{dem-}$ onstrates that few EDU-positive cells were found within the scratch after $48 \mathrm{~h}$ when labeling was performed prior to scratching the cultures. However, cells labeled at $45 \mathrm{~h}$ after scratching ( $3 \mathrm{~h}$ before fixation) showed large numbers of EdU-positive cells in the scratch (fig. 5e), supporting the notion that astrocytes become reactive and hyperproliferative after the scratch insult. This did not occur in the presence of AraC (fig. 5e). The percentage of GFAPpositive cells in the scratch that were also labeled with EdU (fig. 5d) was similar to the percentage of total cells in the scratch labeled with EdU (fig. 5c), indicating no marked differential response of proliferative GFAP-positive cells to the scratch stimulus.

\section{Discussion}

We demonstrated herein that cortical astrocytes derived from rats and mice, two different but closely related species within the family of rodents, show different responses to reactivity-triggering scratch insults in vitro. Cytoskeletal protein expression and intracellular distribution as well as cell morphology and proliferation significantly differed between mouse and rat astrocytes.

The most obvious difference was the markedly decreased percentage of mouse cells that expressed GFAP, compared with rat cells, under basal conditions and in cells responding to scratch wound. However, the mouse cells were astrocytes as they expressed the astrocyte markers GLT1 and S100 $\beta$, and the cultures contained less than $0.5 \%$ microglia and less than $0.1 \%$ oligodendrocytes and neurons (data not shown). Therefore, the lower percentage of cells expressing GFAP in mouse cultures was not due 


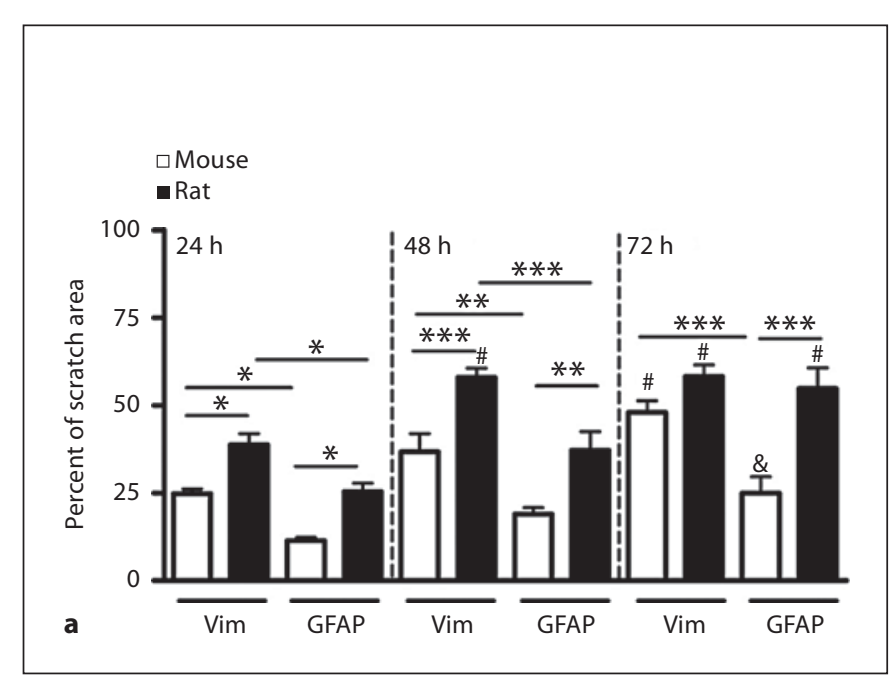

Fig. 4. Comparison of scratch wound closure by vimentin- and GFAP-expressing cells. a Vimentin-expressing cells closed the scratch wound faster than GFAP-expressing cells in both species and rat cells were faster than mouse cells. b The vimentin or GFAP area per cell was determined by dividing the scratch area covered by vimentin or GFAP by the fraction of cells expressing vimentin or GFAP, indicating that mouse cells were larger than rat cells. Results are means \pm SEM of 5 experiments; ${ }^{*} \mathrm{p}<0.05,{ }^{* *} \mathrm{p}<0.01$, ${ }^{* * *} \mathrm{p}<0.001 ;{ }^{\&} \mathrm{p}<0.05$ compared with $24 \mathrm{~h},{ }^{*} \mathrm{p}<0.01$ compared with 24 h. c Immunostaining of GFAP-expressing cells (red) with DAPI-stained nuclei (blue) at $48 \mathrm{~h}$, showing a larger area covered by mouse GFAP-positive cells. Scale bar: $50 \mu \mathrm{m}$.

to differences in culture cell type composition. Given that GFAP is a commonly used marker of astrocytes in cell culture, this may lead to differences in results, depending upon whether rat or mouse cultures are analyzed.

GFAP immunolabeling has been shown to be regulated by posttranslational modification in response to dibutyryl-cAMP, independent of effects at the transcriptional level [23], raising the possibility that different posttranslational modifications in mouse compared with rat cells may contribute to the disparate percentages of astrocytes labeled by the antibody in our experiments. It is also possible that the antibody affinity was lower in mouse astrocytes or that the epitopes on GFAP recognized by the antibody used in these experiments differed somewhat between the species, given slight differences in protein sequence ( $96 \%$ identity). GFAP also binds to multiple proteins [24], and it is possible that the interaction of GFAP with other proteins may differ between species, such that antibody accessibility to epitopes was blocked in some mouse astrocytes. However, we also tested an-
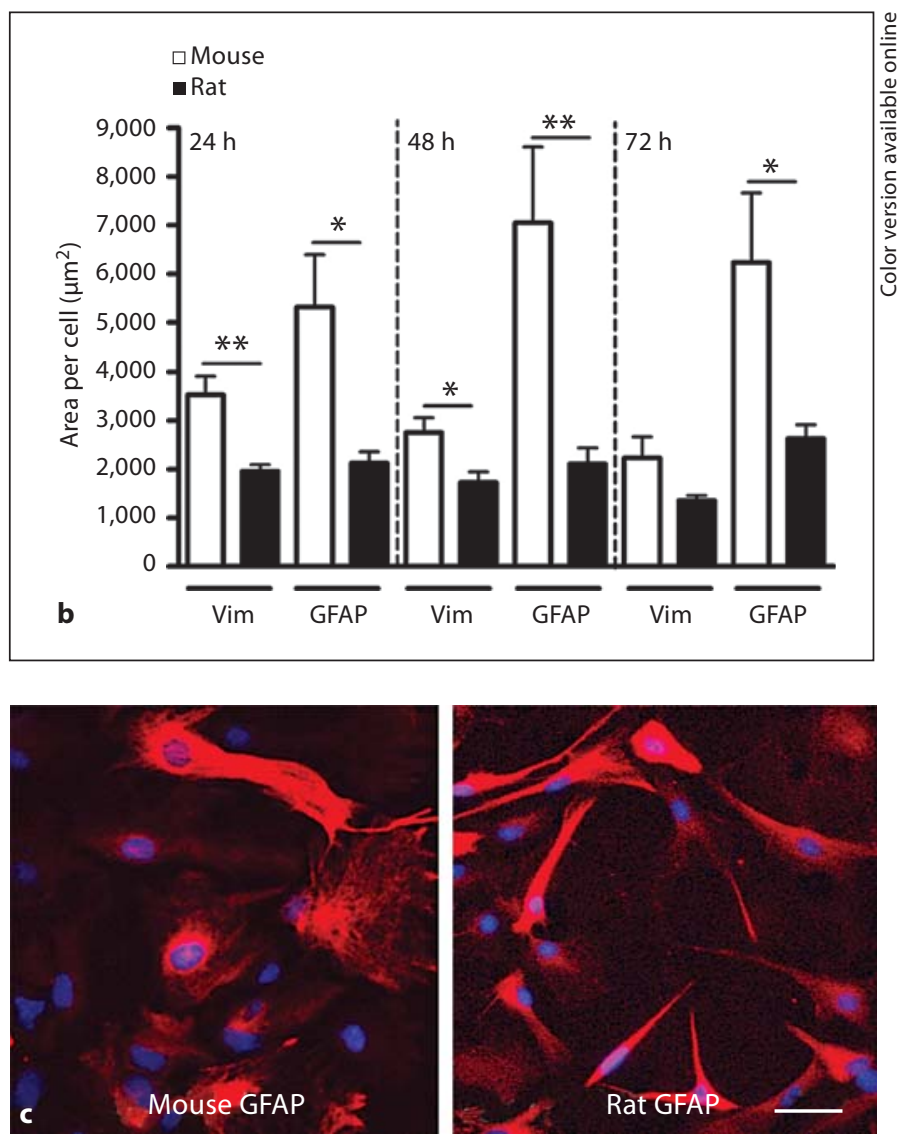

Fig. 5. Inhibition of proliferation ablates the species difference in scratch wound repair. Control cultures (Con) and cultures treated with $\mathrm{AraC}$ for $24 \mathrm{~h}$ prior to and during scratch wound repair were compared for their ability to repair a scratch wound. a AraC inhibited the increase in cell number in the scratch over time in rat and mouse astrocytes. $\mathbf{b} \mathrm{AraC}$ ablated the species differences in the ability of GFAP- and vimentin (Vim)-expressing cells to repair a scratch wound, which was overall decreased in cells of both species. $\mathbf{c E d U}$ was added to cultures for $3 \mathrm{~h}$ prior to scratch wound to label proliferating cells. In control conditions, more rat than mouse EdU-positive cells entered the scratch within $24 \mathrm{~h}$ and this was ablated by AraC addition. $\mathbf{d}$ The percentage of GFAP cells in the scratch that were EdU positive reflected the percentage of total cells that were EdU positive in c. Results are means \pm SEM of 5 experiments; ${ }^{*} \mathrm{p}<0.05,{ }^{* *} \mathrm{p}<0.01,{ }^{* * *} \mathrm{p}<0.001$. e Immunostaining for GFAP and EdU in rat and mouse scratches at $48 \mathrm{~h}$ after scratching, with nuclei stained by DAPI. Top panels for each condition show merged images, with EdU staining alone shown below for clarity. Few cells that were proliferative prior to scratching (EdU positive) entered the scratch; however, many cells within the scratch were proliferative when EdU was added $3 \mathrm{~h}$ prior to fixation ( $45 \mathrm{~h}$ after scratching), which was ablated by AraC treatment. Scale bar: $150 \mu \mathrm{m}$. 


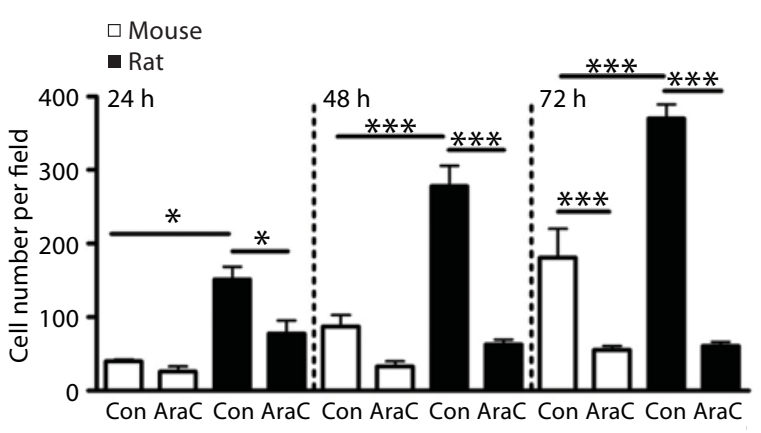

a
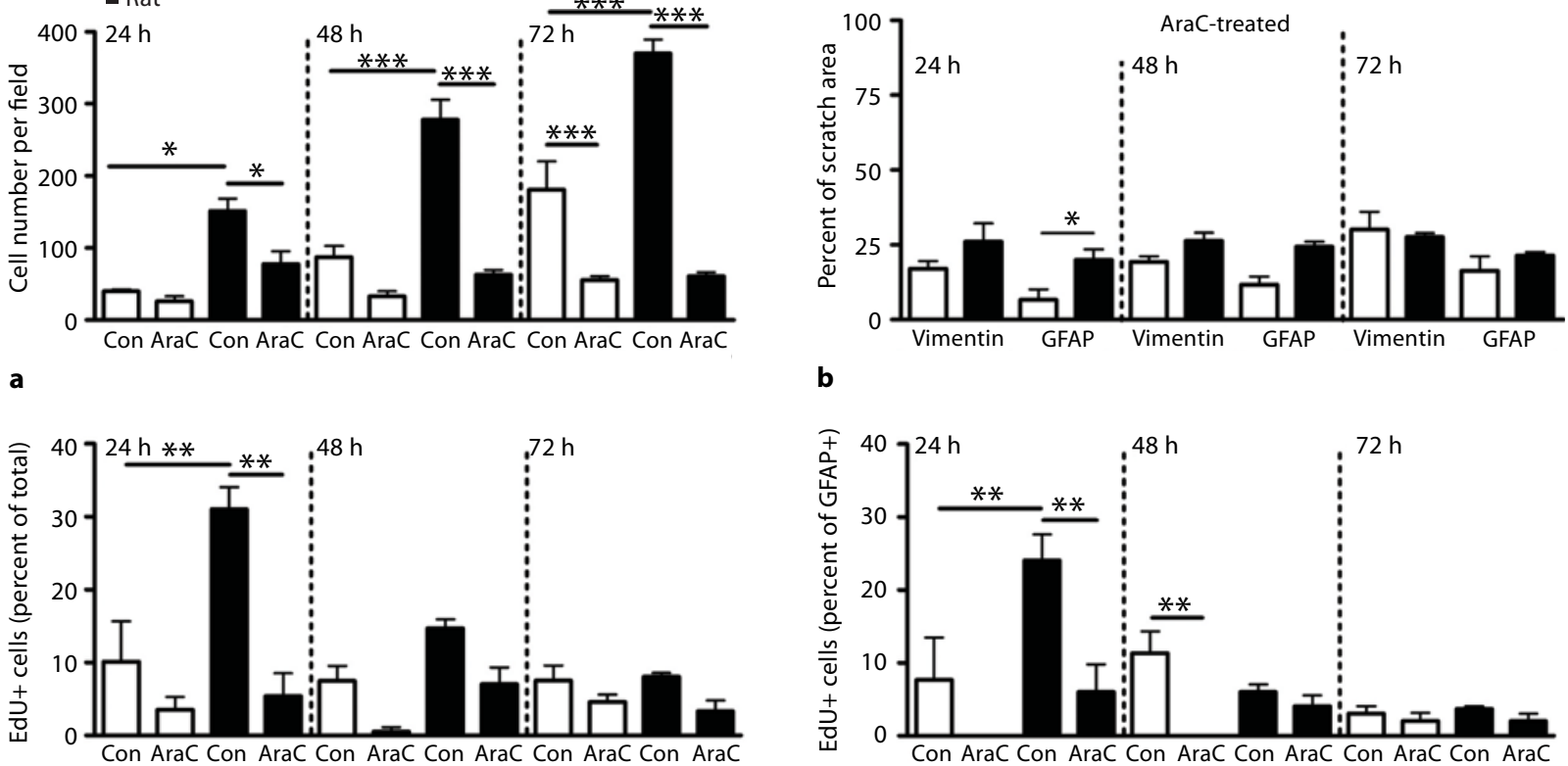

b
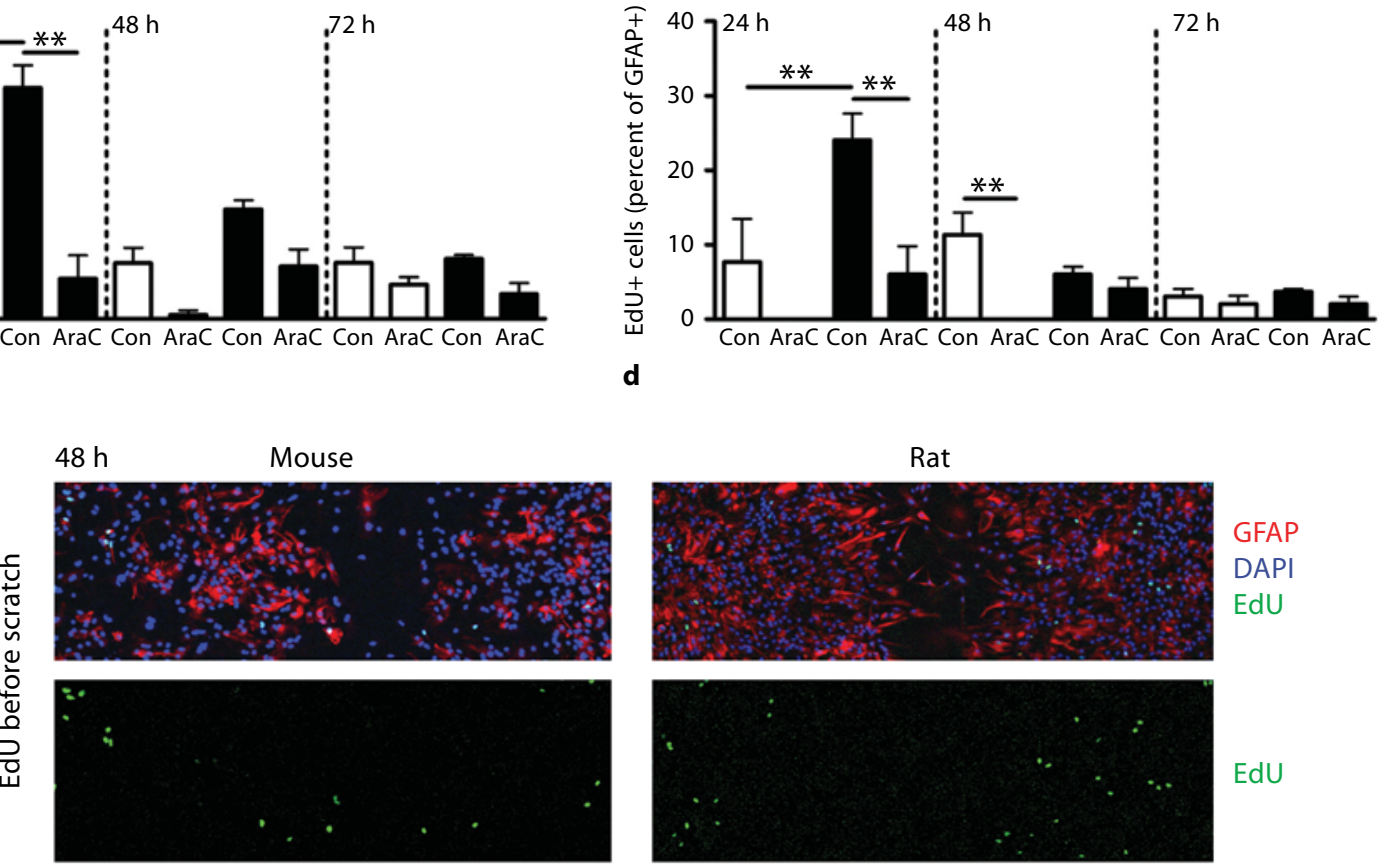

GFAP

DAPI

EdU
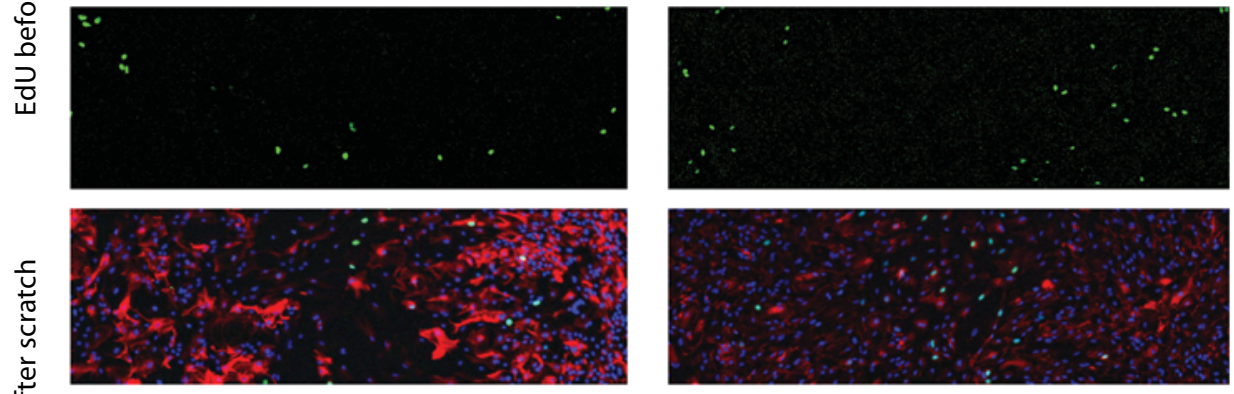

GFAP

DAPI

EdU
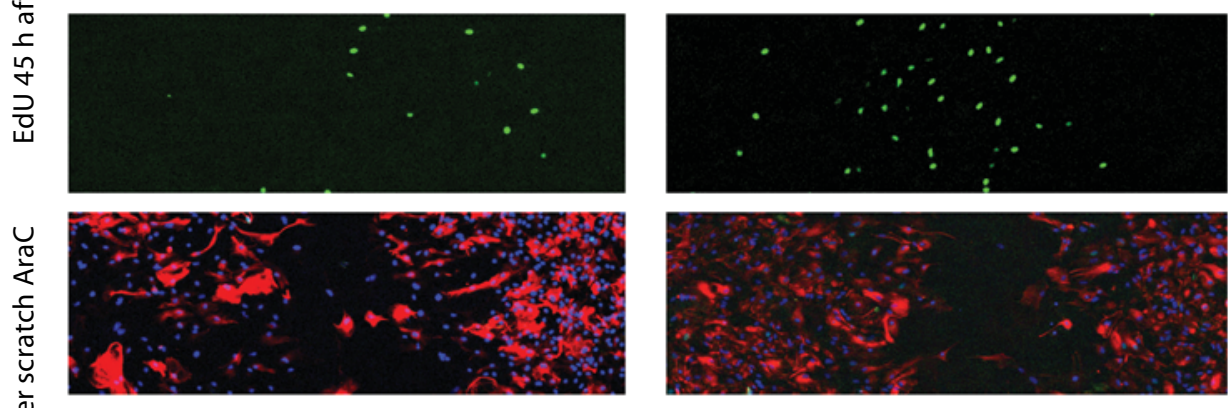

GFAP

DAPI

EdU

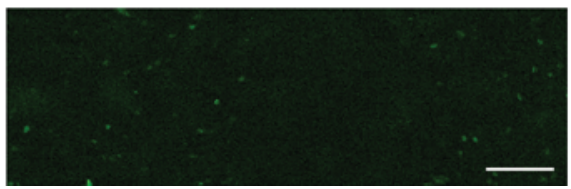


other commonly used polyclonal GFAP antibody (rabbit anti-cow GFAP from Dako, online suppl. fig. 1), which gave similar results to the mouse anti-GFAP antibody, so epitope accessibility or affinity is not likely to be the cause of the detected difference in cell numbers expressing GFAP between species.

The difference in the molecular size of the GFAP protein determined by Western blot analysis is probably due to a slightly different protein sequence. Although there are several isoforms, most are approximately 428 (mouse) to 430 (rat) amino acids in length and a protein sequence blast search between the two sequences shows up to $96 \%$ protein sequence identity. This small difference between the two protein sequences might account for some of the observed differences in the molecular weight, but the differences are more likely due to differences in posttranslational modification. There are multiple phosphorylation sites in GFAP, and mouse GFAP has 3 fewer phosphorylation sites ( 1 serine and 2 threonine) than rat GFAP based on a blast search comparison [25].

We could not detect any changes in GFAP or vimentin levels at 2 days after the scratch wound procedure by Western blot analysis, as previously reported in scratch wound experiments conducted with spinal cord astrocytes of rats [26]. The fact that we failed to detect GFAP upregulation may be explained by the localized cytoskeletal protein upregulation at the border of the scratch, which does not significantly change the overall expression levels, especially since the newborn proliferative astrocytes within the scratch are mostly GFAP negative.

Vimentin, like GFAP, is an intermediary filament that is upregulated in reactive astrocytes after a CNS insult [27]. It is also known to be expressed in immature astrocytes and astrocyte precursors [28-30] and decreases over time after P14, concomitantly with the increase in GFAP expression after P7 [31, 32]. In our in vitro experiments, we saw similarly increased expression of vimentin by $24 \mathrm{~h}$ after scratching in astrocytes bordering the scratch site, confirming vimentin as a reactive astrocyte marker. Similar vimentin upregulation by mouse and rat astrocytes has recently been observed in vivo following mild cerebral contusion [33]. Experiments with GFAP-/animals have shown that astrocytes in the corpus callosum and hippocampus are unable to form proper filaments in the absence of GFAP, while astrocytes from vimentin-/- animals showed abnormal GFAP filament formation, suggesting a strong binding partnership between these two intermediate filaments [34].

Unexpectedly, although there were differences in cytoskeletal protein expression and cellular morphology between astrocytes of the two species, their differential ability to respond to the scratch wound largely seemed to rely on their proliferative capacity. Previous research has shown that astrocytes in a scratch wound model react with increased proliferation, as measured by BrdU uptake [12] and it has further been suggested that in high-density cultures astrocytes exhibit homotypic cellcell contact inhibition of proliferation. After injury or insult in the scratch wound model, the cell-cell contact inhibition of proliferation is released and cells become hyperproliferative $[12,20]$. Data presented here show that proliferation was significantly increased in cortical astrocytes of both species within the scratch compared with cells in control areas next to the scratch, demonstrating that, as expected, the scratch insult caused astrocyte reactivity. Interestingly, the proliferation rate in rat astrocytes was much higher than in mouse cells, suggesting that intrinsic kinetic differences in proliferation exist between cells of the two species in response to scratch insult. Concomitantly with the increased proliferation, rat astrocytes closed a scratch wound faster than mouse astrocytes. Blocking proliferation by the use of AraC supported the suggestion that the faster scratch wound closure by rat cells was due to increased proliferation, not migration, since no species differences in extent of scratch closure were found after AraC treatment.

Although rat cells closed the scratch slightly faster due to enhanced proliferation, mouse cells were also able to repair the scratch by $72 \mathrm{~h}$, even though there were fewer cells. Based on cytoskeletal protein expression, the mouse cells that invaded the scratch appeared to be larger than the rat cells, such that fewer cells could fill the same area. Overall, the end result of scratch wound closure was achieved by astrocytes of both species, as would be expected, but the mechanisms by which they did so differed. Cortical astrocyte reactivity in mice in vivo also appears to be mediated primarily by hypertrophy rather than by proliferation. Following stab wound, astrocytes bordering a cortical lesion were found to upregulate GFAP and become hypertrophic, without increasing proliferation [35], similar to our observed in vitro results.

In this study, we have compared cortical gray matter astrocytes of relatively closely related species and found significant differences in a number of aspects of their basic biology. Whether there are similar species differences between white matter astrocytes remains to be determined. It may be expected that there would be little difference in GFAP expression in white matter astrocytes as, unlike gray matter astrocytes, they maintain high levels of GFAP expression in adulthood [36, 37]. It will also be 
of interest to compare astrocyte responses between other species, including human and common laboratory species such as rodents, to assess whether results obtained by analysis of rodent cells are generally applicable to cells from other species.

\section{Acknowledgment}

A.M.T. is supported by a fellowship from the National Health and Medical Research Council of Australia (ID No. 628344).

\section{References}

-1 Pekny M, Nilsson M: Astrocyte activation and reactive gliosis. Glia 2005;50:427-434.

$>2$ Nieto-Sampedro M: Neurite outgrowth inhibitors in gliotic tissue. Adv Exp Med Biol 1999;468:207-224.

$>3$ Stichel CC, Muller HW: The CNS lesion scar: new vistas on an old regeneration barrier. Cell Tissue Res 1998;294:1-9.

$\checkmark 4$ Abd-El-Basset EM, Fedoroff S: Upregulation of $\mathrm{F}$-actin and $\alpha$-actinin in reactive astrocytes. J Neurosci Res 1997;49:608-616.

5 Eng LF, Ghirnikar RS: GFAP and astrogliosis. Brain Pathol 1994;4:229-237.

$\checkmark 6$ Ciesielski-Treska J, Guerold B, Aunis D: Immunofluorescence study on the organization of actin in astroglial cells in primary cultures. Neuroscience 1982;7:509-522.

7 Oey J: Noradrenaline-refractoriness in cultured glioma cells. Exp Brain Res 1976;25: 359-368.

$>8$ Fedoroff S, McAuley WA, Houle JD, Devon RM: Astrocyte cell lineage. V. Similarity of astrocytes that form in the presence of $\mathrm{dBcAMP}$ in cultures to reactive astrocytes in vivo. J Neurosci Res 1984;12:14-27.

$>9$ Wandosell F, Bovolenta P, Nieto-Sampedro M: Differences between reactive astrocytes and cultured astrocytes treated with di-butyryl-cyclic AMP. J Neuropathol Exp Neurol 1993;52:205-215.

10 Hou YJ, Yu AC, Garcia JM, Aotaki-Keen A, Lee YL, Eng LF, Hjelmeland LJ, Menon VK: Astrogliosis in culture. IV. Effects of basic fibroblast growth factor. J Neurosci Res 1995; 40:359-370.

$\checkmark 11$ Yu AC, Lee YL, Eng LF: Astrogliosis in culture. I. The model and the effect of antisense oligonucleotides on glial fibrillary acidic protein synthesis. J Neurosci Res 1993;34: 295-303.

-12 Kornyei Z, Czirok A, Vicsek T, Madarasz E: Proliferative and migratory responses of astrocytes to in vitro injury. J Neurosci Res 2000;61:421-429.

$>13$ Frisen J, Johansson CB, Torok C, Risling M, Lendahl U: Rapid, widespread, and longlasting induction of nestin contributes to the generation of glial scar tissue after CNS injury. J Cell Biol 1995;131:453-464.

$\checkmark 14$ Nishio T, Kawaguchi S, Yamamoto M, Iseda $\mathrm{T}$, Kawasaki T, Hase T: Tenascin-C regulates proliferation and migration of cultured astrocytes in a scratch wound assay. Neuroscience $2005 ; 132: 87-102$.
15 Puschmann TB, Turnley AM: Eph receptor tyrosine kinases regulate astrocyte cytoskeletal rearrangement and focal adhesion formation. J Neurochem 2010;113:881-894.

16 Faber-Elman A, Solomon A, Abraham JA, Marikovsky M, Schwartz M: Involvement of wound-associated factors in rat brain astrocyte migratory response to axonal injury: in vitro simulation. J Clin Invest 1996;97:162171.

17 Savani RC, Wang C, Yang B, Zhang S, Kinsella MG, Wight TN, Stern R, Nance DM, Turley EA: Migration of bovine aortic smooth muscle cells after wounding injury. The role of hyaluronan and RHAMM. J Clin Invest 1995;95:1158-1168.

$>18$ Elner SG, Elner VM, Pavilack MA, Davis HR, Cornicelli JA, Yue BY: Human and monkey corneal endothelium expression of lowdensity lipoprotein receptors. Am J Ophthalmol 1991;111:84-91.

19 Di Giovanni S, Movsesyan V, Ahmed F, Cernak I, Schinelli S, Stoica B, Faden AI: Cell cycle inhibition provides neuroprotection and reduces glial proliferation and scar formation after traumatic brain injury. Proc Natl Acad Sci USA 2005;102:8333-8338.

20 Nakatsuji Y, Miller RH: Density dependent modulation of cell cycle protein expression in astrocytes. J Neurosci Res 2001;66:487496.

21 Schwartz JP, Nishiyama N: Neurotrophic factor gene expression in astrocytes during development and following injury. Brain Res Bull 1994;35:403-407.

22 Wu VW, Schwartz JP: Cell culture models for reactive gliosis: new perspectives. J Neurosci Res 1998;51:675-681.

23 Le Prince G, Fages C, Rolland B, Nunez J, Tardy M: DBcAMP effect on the expression of GFAP and of its encoding mRNA in astroglial primary cultures. Glia 1991;4:322-326.

24 Sullivan SM, Lee A, Bjorkman ST, Miller SM, Sullivan RK, Poronnik P, Colditz PB, Pow DV: Cytoskeletal anchoring of GLAST determines susceptibility to brain damage: an identified role for GFAP. J Biol Chem 2007; 282:29414-29423.

-25 Altschul SF, Madden TL, Schaffer AA, Zhang J, Zhang Z, Miller W, Lipman DJ: Gapped BLAST and PSI-BLAST: a new generation of protein database search programs. Nucleic Acids Res 1997;25:3389-3402.
26 Yang H, Cheng XP, Li JW, Yao Q, Ju G: Dedifferentiation response of cultured astrocytes to injury induced by scratch or conditioned culture medium of scratch-insulted astrocytes. Cell Mol Neurobiol 2009;29:455473 .

27 Eddleston M, Mucke L: Molecular profile of reactive astrocytes - implications for their role in neurologic disease. Neuroscience 1993;54:15-36.

28 Schnitzer J, Franke WW, Schachner M: Immunocytochemical demonstration of vimentin in astrocytes and ependymal cells of developing and adult mouse nervous system. J Cell Biol 1981;90:435-447.

29 Bignami A, Raju T, Dahl D: Localization of vimentin, the nonspecific intermediate filament protein, in embryonal glia and in early differentiating neurons. In vivo and in vitro immunofluorescence study of the rat embryo with vimentin and neurofilament antisera. Dev Biol 1982;91:286-295.

30 Lendahl U, Zimmerman LB, McKay RD: CNS stem cells express a new class of intermediate filament protein. Cell 1990;60:585595.

31 Bovolenta P, Liem RK, Mason CA: Development of cerebellar astroglia: transitions in form and cytoskeletal content. Dev Biol 1984;102:248-259.

32 Pixley SK, de Vellis J: Transition between immature radial glia and mature astrocytes studied with a monoclonal antibody to vimentin. Brain Res 1984;317:201-209.

33 Ekmark-Lewen S, Lewen A, Israelsson C, Li GL, Farooque M, Olsson Y, Ebendal T, Hillered L: Vimentin and GFAP responses in astrocytes after contusion trauma to the murine brain. Restor Neurol Neurosci 2010;28: 311-321.

34 Pekny M, Lane EB: Intermediate filaments and stress. Exp Cell Res 2007;313:22442254.

35 Burns KA, Murphy B, Danzer SC, Kuan CY: Developmental and post-injury cortical gliogenesis: a genetic fate-mapping study with Nestin-CreER mice. Glia 2009;57:1115-1129.

-36 Landry CF, Ivy GO, Brown IR: Developmental expression of glial fibrillary acidic protein mRNA in the rat brain analyzed by in situ hybridization. J Neurosci Res 1990;25:194203.

37 Lewis SA, Cowan NJ: Temporal expression of mouse glial fibrillary acidic protein mRNA studied by a rapid in situ hybridization procedure. J Neurochem 1985;45:913-919. 